\title{
Oralités de résistance et oralités joyeuses
}

Oralities of resistance and joyful oralities

\section{Marie-Christine Vinson}

\section{OpenEdition}

Journals

Édition électronique

URL : https://journals.openedition.org/clo/8012

DOI : $10.4000 /$ clo. 8012

ISSN : 2266-1816

Éditeur

INALCO

\section{Édition imprimée}

Date de publication : 3 décembre 2019

Pagination : 105-139

ISBN : 9782858313716

ISSN : 0396-891X

\section{Référence électronique}

Marie-Christine Vinson, "Oralités de résistance et oralités joyeuses », Cahiers de littérature orale [En ligne], 86 | 2019, mis en ligne le 03 décembre 2020, consulté le 25 mars 2022. URL : http:// journals.openedition.org/clo/8012; DOI : https://doi.org/10.4000/clo.8012

\section{(c) $(1) \Theta$}

Cahiers de littérature orale est mis à disposition selon les termes de la Licence Creative Commons Attribution - Pas d'Utilisation Commerciale 4.0 International. 


\title{
Oralités de résistance et oralités joyeuses
}

\author{
Marie-Christine Vinson \\ Université de Lorraine - CREM
}

Dans la soirée la salle devient un peu houleuse. Il y a une nouvelle bande de garçons qui s'amusent et qui ne comprennent pas évidemment ce qu'est la bibliothèque.

Cette notation en date du 28 novembre 1924 se trouve sur la sixième page du Journal que tient Claire Huchet. Ce journal commence le 12 novembre 1924, date à laquelle a eu lieu l'inauguration de L'Heure joyeuse, première bibliothèque pour enfants ${ }^{1}$ créée à Paris au 3 rue Boutebrie (Quartier Latin). Le 6 décembre 1934, Jacqueline Dreyfus², formée par les bibliothécaires de L'Heure joyeuse, note de

\footnotetext{
1. Après la Première Guerre mondiale, le Book Committee on Children's Libraries, présidé par Caroline Griffiths, a le projet éducatif de créer des lieux de culture où les enfants seront éduqués dans un esprit pacifique. La bibliothèque pour enfants en offrant des livres au plus grand nombre doit permettre de construire cet avenir tourné vers la paix, elle doit contribuer à la mise en place d'une compréhension et d'une entente entre les peuples. Aussi le comité propose-t-il aux municipalités de Bruxelles et de Paris une bibliothèque de prêt gratuite, avec une salle de lecture, uniquement destinée aux enfants. La première Heure joyeuse ouvre le 24 septembre 1920 à Bruxelles. Le 12 novembre 1924 est inaugurée, 3 rue Boutebrie, la première bibliothèque consacrée à la jeunesse de France, baptisée elle aussi Heure joyeuse.

2. Jacqueline Dreyfus a commencé par animer une bibliothèque à Ménilmontant. Après une formation à L'Heure joyeuse et des études en pédagogie avec Henri Wallon, elle fut nommée bibliothécaire à la section jeunesse de la bibliothèque de la rue Fessart de 1934 à 1937.
} 


\section{CAHIERS DE LITTÉRATURE ORALE}

son côté, à propos de l'Heure du conte qui se déroule à la bibliothèque de la rue Fessart $^{3}$ dans le $19^{\mathrm{e}}$ arrondissement :

Le grand nombre des lecteurs nous empêche de sentir les réactions individuelles et le public n'est pas encore assez formé pour que nous sentions une communion entre lui et nous.

Ces deux remarques laissent deviner les difficultés que rencontrent dans leur travail ces premières bibliothécaires pour la jeunesse. Il faut en effet former dans la salle de lecture des lecteurs intéressés par les livres et actifs dans la communauté des lecteurs ; et lors des heures du conte, il s'agit cette fois de former des auditeurs à être attentifs à la performance de la conteuse mais aussi qui participent aux mondes imaginaires qui leur sont offerts de vive voix.

Ces propositions culturelles - devenir lecteurs, devenir auditeurs suscitent chez les enfants et les adolescents concernés des résistances fortes et des compromis mais aussi, dans certains cas, des adhésions enthousiastes. Ces résistances à une acculturation écrite - perçue comme lettrée - prennent souvent la forme d'oralités juvéniles plus ou moins éruptives ${ }^{4}$. Ces oralités contestataires ou indisciplinées font ainsi irruption dans le monde de la culture écrite cultivée qui règne à la bibliothèque. Elles en bousculent l'ordre voire en carnavalisent symboliquement les règles :

Tout le monde travaille avec calme et lit avec intérêt jusqu'à six heures où Fersancourt hurle dans la bibliothèque ${ }^{5}$.

\footnotetext{
3. En 1922 le Comité américain pour les régions dévastées (CARD) crée, au cœur de Belleville sur un terrain de la ville de Paris, une bibliothèque. Installée, au début, dans un baraquement provisoire au 6 de la rue Fessart, cette bibliothèque est la première à pratiquer à Paris le libre accès (classification Dewey des ouvrages) et à offrir aux enfants une section spéciale. Dix ans plus tard la bibliothèque Fessart emménage dans le bâtiment à façade de briques où elle se trouve aujourd'hui.

4. On entend « oralité » au sens de l'anthropologie linguistique et culturelle. L'oral des linguistes n'est donc qu'une des manifestations de l'oralité. Ainsi la «culture orale » des jeunes enfants est-elle ici volontiers publique et collective alors que la « culture écrite » proposée par les livres tend à être plus secrète et plus personnelle. À ce silence des livres s'opposent donc en quelque façon les bruits et les cris, les rumeurs et les humeurs, les applaudissements et les éclats de voix, l'écoute attentive et l'approbation muette, bref les mille et une formes de l'expressivité des corps et de la sonorité de la langue.
}

5. Huchet, Journal 1924-1925, 29 octobre 1925, p. 121. 


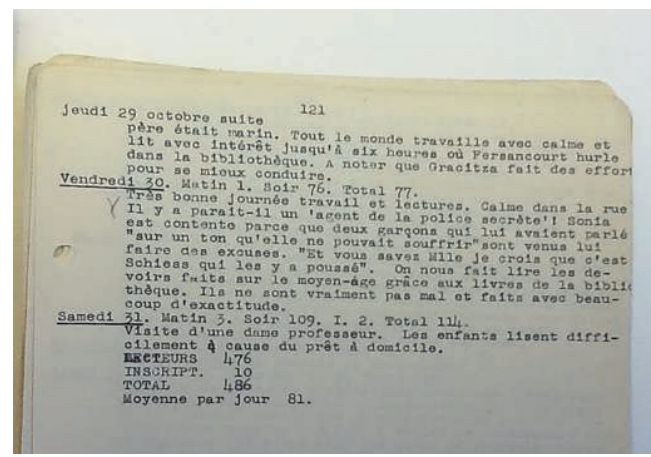

Illustration 1

Claire Huchet, Journal 1924-1925 - 29 octobre 1925.

Fonds patrimonial Heure joyeuse

Ce cri rompt le pacte de calme, de silence, de discrétion et de retenue propre à la salle de lecture. Cet abandon du corps lisant et cette irruption d'un corps hurlant qui s'ensauvage sans retenue aux yeux de tous montre le difficile travail sur soi de discipline sociale et culturelle.

Lucienne Meyer et les Touzelet ne reprennent pas de livres pendant quelques temps, elles ont trop de devoirs. Viennent 2 ou 3 fois, ouvrent la porte, hurlent et se sauvent ${ }^{6}$.

Là encore, des cris protestataires entrent par effraction dans un espace de culture où les corps sont pour les uns domestiqués, civilisés pour les autres. Ces formes d'oralités ensauvagées - signes publics d'indiscipline et d'insubordination - se donnent à entendre avec une certaine insistance. Elles se manifestent aussi bien dans la salle de lecture que lors de l'Heure du conte. Elles sont présentes à L'Heure joyeuse comme à la bibliothèque de Ménilmontant ou à celle de la rue Fessart.

\section{Salle de lecture et communauté des lecteurs}

[...] la porte de la rue s'ouvre sur un tambour muni d'une porte de chaque côté et où une inscription invite les enfants à s'essuyer les pieds. En face de l'entrée est le bureau de la bibliothécaire où

6. Dreyfus, Comptes rendus de la bibliothèque de Ménilmontant, 6 février 1932. 
se trouvent tout ouverts le registre d'inscription et la feuille de présence $^{7}$.

Tout un monde de culture est donné à voir dans cette description de l'entrée de L'Heure joyeuse faite par Henri Lemaître lors de l'inauguration. Et s'il est un endroit de la bibliothèque particulièrement intéressant pour observer ces attitudes gouailleuses et agressives dont nous venons de parler, c'est la porte.

Frontière entre la rue et la salle de lecture, la porte est bien souvent le lieu scénique où se jouent des affrontements multiples. On le sait, la porte est souvent « le marqueur d'une limite essentielle dans l'ordre du monde, celle opposant le "nous" de la communauté et les autres, les étrangers, l'inconnu ${ }^{8} \gg$. Aussi les anthropologues définissent-ils ses limites comme « des lieux de lutte ${ }^{9} »$.

S'il n'est pas question de comparer ici le passage du seuil de la bibliothèque à un rite de passage, force est de constater que son franchissement symbolique et physique ne va pas de soi. Le quartier de la rue Boutebrie est socialement diversifié. À la bibliothèque se côtoient de jeunes employés, des ouvriers, des apprentis mais aussi des écoliers, des lycéens ; des petits « misérables » issus de milieu populaire comme des jeunes lecteurs de la classe moyenne ou de la bourgeoisie ${ }^{10}$. La cité de Ménilmontant est plutôt ouvrière, le quartier de la rue Fessart à dominante

7. Lemaitre, 1925, p. 38-39.

8. KaufMan, 1996, p. 280.

9. Bourdieu, 1980, p. 374.

10. Dans une conférence faite lors de journées d'études organisées par La Joie par les livres en 1985, Marguerite Gruny rappelle la sociologie du quartier dans lequel se trouvait la rue Boutebrie et la bibliothèque : « Le quartier Saint-Séverin, avant la guerre, n'avait pas du tout l'aspect cosmopolite et touristique d'aujourd'hui : les maisons du XVII et $\mathrm{du} \mathrm{XVIII}^{\mathrm{e}}$ siècle, restaurées et transformées depuis, abritaient une population en général peu aisée qui contrastait avec la bourgeoisie intellectuelle des boulevards SaintGermain et Saint-Michel. Or dans ces milieux d'artisans, d'employés modestes, de petits commerçants, bien rares étaient les ménagères disposées à acheter des livres comme on en voit aujourd'hui dans les magasins et les grandes surfaces; les livres représentaient pour elles un luxe - inutile même - et leurs enfants devaient se contenter d'illustrés qu'ils se repassaient entre eux. Aussi, pour les démunis avides de lecture, quelle joie d'entrer à L'Heure joyeuse ! », GRUnY, 1986, p. 51-52. Dans son livre Les livres, les enfants et les hommes, paru en 1932, Paul Hazard décrit ainsi la rue Boutebrie : « Le jour où votre promenade vous mènera vers le vieux quartier qui entoure Sant-Séverin, allez voir la bibliothèque que les Américains ont organisé pour les petits Français et pour les étrangers qui habitent les parages, Orientaux, Russes, Polonais ; à peu près toutes les nations sont représentées dans ce fouillis de maisons lépreuses, dans ces ruelles qui évoquent le temps 
populaire. L'acceptation d'une institution de lecture nouvelle et spécifique, $a$ fortiori son appropriation libre et responsable ne va pas de soi.

\section{...rester à la porte}

Il n'est pas facile pour tout le monde de faire le premier pas et de pénétrer dans la salle. Bien souvent la porte, avant même son passage, est un lieu où de fortes tensions peuvent s'exprimer, violemment et quotidiennement. Or, quelle meilleure manifestation de contre-culture ou de contestation culturelle du temple de l'écriture distinguée que l'expression d'oralités plébéiennes protestataires et d'obstructions physiques :

À trois heures des masses de lecteurs auxquels se joignent quelques voyous, encombrent l'escalier. Le gardien est blanc de colère et menace de mettre bibliothèque et bibliothécaires à la porte. [...] À quatre heures nous ouvrons la porte aux lecteurs, renvoyons les petits frères et sœurs, les mains sales, les sans-papiers ${ }^{11}$.

Une autre fois, c'est une petite bande de gamins du quartier qui essaie d'opposer par corps et par cris la culture de rue, agressive, populaire et machiste, au libre chemin vers la culture écrite :

Après $17 \mathrm{~h}$, il y a du bruit dans la rue. Des gamins font tomber une fillette qui rentre à la bibliothèque. Ils grimpent sur les fenêtres et poussent divers cris. Il faudra un agent (de police) pour leur faire un petit peu peur ${ }^{12}$.

Les sauvageons font donc de l'obstruction. Éloignés des livres, récalcitrants à la lecture instituée et peut-être associée à l'école - la journée vient juste de se terminer -, ils se refusent à passer le pas. Ils prennent plaisir à effrayer ceux qui tentent de franchir le seuil et à provoquer les adultes qui de l'autre côté de la porte doivent faire respecter l'ordre... en appelant les forces de l'ordre, au besoin. Les agents sont ainsi sollicités plusieurs fois :

Bruit à la porte. Kroeger va chercher un agent ${ }^{13}$.

où Villon y hantait les bouges et cabarets. La rue a gardé son vieux nom savoureux : c'est la rue Boutebrie. », HAZARD, 1967, p. 113.

11. Dreyfus, Comptes rendus de la bibliothèque de Ménilmontant, 11 novembre 1931.

12. Huchet, Journal 1924-1925, 13 novembre 1924, p. 1.

13. Ibid., 8 octobre 1925, p. 114. 
Il y a du bruit à la porte et la salle s'en ressent. Le commissaire ne pourra envoyer un agent que dans quelques jours ${ }^{14}$.

Il y a un agent à la porte et on est tranquille ${ }^{15}$.

Bien sûr, ce recours uniquement répressif n'est pas la solution recherchée et constitue un véritable aveu d'impuissance :

Salle pleine le soir. Lecture attentive. Ils ne se laissent pas distraire par le bruit que font deux gamins à la porte. Il n'y a pas de sergent de ville. Et d'ailleurs le sergent de ville est-il une solution ${ }^{16}$ ?

Après la fermeture, un galopin qui était venu en novembre (on est en mars) à la bibliothèque frappe à coups redoublés à la fenêtre. La fenêtre s'ouvre et un pot de jacinthe s'effondre sur le sol. Il n'y a pas d'agent naturellement. J'ai menacé le galopin de la police et ce n'est sans doute pas ce qu'il aurait fallu faire. J'aurais dû le faire entrer gentiment. On n'a pas toujours la patience nécessaire ${ }^{17}$.

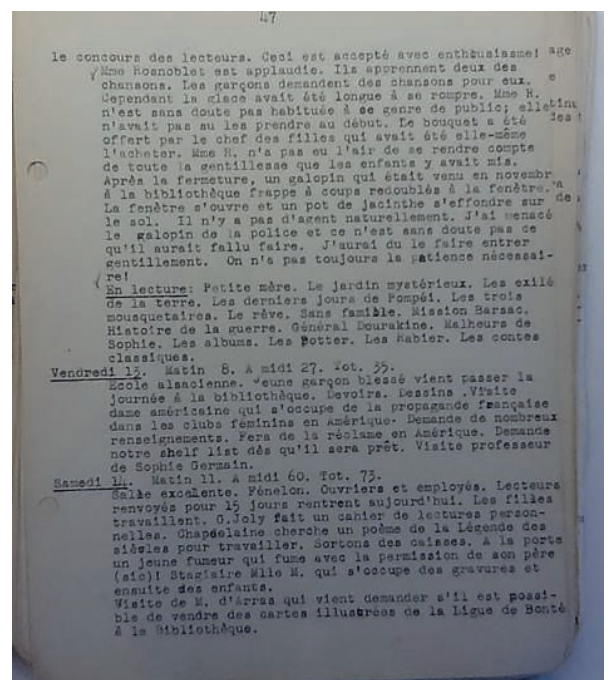

\section{Illustration 2 \\ Claire Huchet, Journal 1924-1925, \\ 12 mars 1925}

Fonds patrimonial Heure joyeuse

14. Ibid., 14 octobre, p. 116.

15. Huchet, Journal 1925-1926, 27 janvier 1926, p. 20.

16. Huchet, Journal 1924-1925, 19 janvier 1925, p. 25.

17.Ibid., 12 mars 1925, p. 47. 
Notre bibliothécaire, « véritable petit dieu de seuil ${ }^{18}$ », sait pertinemment qu'il faut de l'empathie pour convaincre certains enfants de franchir le pas et d'entrer dans le monde distinctif des lecteurs. En juillet de la même année, Claire Huchet écrit :

Un garçon non inscrit fait du bruit à la porte de la bibliothèque ; on essaie de lui parler ; il confie qu'il se couche tous les jours à 2 heures du matin ; on lui conseille de venir s'inscrire comme lecteur ${ }^{19}$.

Il arrive aussi que la bibliothécaire (ici Jacqueline Dreyfus) anticipe un recours au représentant de l'ordre qui s'avère injustifié : « Entrée et sortie calmes; l'agent demandé par téléphone n'est pas venu ${ }^{20}$. »

\section{... être mis à la porte}

La porte, cet entre-deux qui sépare la rue de la salle de lecture, est aussi une limite entre un monde connu (le quartier) et un monde nouveau plus ou moins attractif ou répulsif (la bibliothèque jeunesse), limite dont le franchissement peut être frappé d'interdit(s). À ce titre il est inévitable que la porte d'entrée cristallise de vives réactions. Ainsi, si certains n'ont aucune envie d'entrer ou même essaient d'en dissuader leurs pairs, d'autres sont contraints de sortir. En effet on peut aussi être mis à la porte... Quand un enfant passe la porte et pénètre pour la première fois dans la bibliothèque, il se doit de prendre un engagement en s'inscrivant sur le registre d'entrée :

En écrivant mon nom dans ce livre, je deviens membre de L'Heure joyeuse, et promets de prendre soin des livres et d'aider le bibliothécaire à rendre notre bibliothèque agréable et utile à tous.

La promesse écrite est un contrat moral. Elle oblige. Il est donc interdit de faire un usage mal approprié d'un document ou plus ordinairement sans doute de troubler le calme sans se manquer à soi-même :

18. BACHELARD, 1994, p. 200.

19. Huchet, Journal 1924-1925, 27 juillet 1925, p. 98.

20. Dreyfus, Comptes rendus de la bibliothèque de Ménilmontant, 24 décembre 1932. 
Nous renvoyons Bernard N. sans livre parce qu'il parle à haute voix après un speach que nous avons fait aux enfants en leur priant de ne pas lancer les albums sur les rayons, de mieux se tenir, etc. ${ }^{21}$

Le jeune Bernard résistera malgré tout. Il fera fi des demandes perçues comme des injonctions contraignantes; il refusera le parler bibliothéque - parler « doucement »- et continuera à parler à haute voix, exactement comme s'il était dans la rue. Ce non-respect de la consigne relève-t-il de l'incompréhension ou de l'insubordination ? Des deux peut-être. Ces oralités de résistances montrent clairement le chemin social et culturel que certains doivent parcourir pour accepter puis éventuellement intérioriser des règles de comportement qui leur sont largement étrangères et qu'ils trouvent étranges en somme :

Il faut calmer Bilman et Moreau. L'un d'eux : « C'est une église ici ! » avec un accent inimitable ${ }^{22}$.

L'église est la sacro-sainte référence mobilisée pour évoquer le silence respectueux et presque scripturaire qui semble tenir à distance les profanes : là tout n'est qu'ordre et méditation, calme et discrétion. À l'opposé de la vie de la rue parisienne et de sa socialité active, expressive, orale. Et l'accent « inimitable » des gamins des faubourgs qui fait irruption dans l'enceinte de la bibliothèque ce temple des livres - manifeste chez la bibliothécaire la reconnaissance d'une altérité sociale et signifie sans doute dans le même temps le travail de civilisation des mœurs et des langages à accomplir.

En effet, les bibliothécaires ne se présentent ni ne se prétendent des « ennemis ». Elles cherchent au contraire « à inspirer confiance petit à petit » et aident à s'approprier les us et coutumes qui conviennent. Elles savent par expérience quotidienne que cette acculturation est un ajustement sinon une contention du corps - sa tenue et sa retenue - aux usages lettrés qui « sera peut-être $\operatorname{long}^{23} \gg$ :

[...] 4 gamins de 14 à 15 ans. De vrais loustics, pas méchants mais farceurs comme des Parisiens. Ils lisent sans broncher jusqu'à 16h. À $16 \mathrm{~h}$ ils émettent quelques cris. Leur conseille d'aller se

21. Ibid., 2 janvier 1932.

22. Ibid., 17 février 1932.

23. Huchet, Journal 1924-1925, $1^{\text {er }}$ décembre 1924, p. 7. 
dégourdir les jambes. Ils le font de bonne grâce et reviennent une demie heure après, calmés ${ }^{24}$.

[...] nous mettons à la porte $[. .$.$] Loulou que nous persuadons$ avec assez de mal de rentrer chez lui ${ }^{25}$.

Mais si conseils, remontrances et autres gronderies ne servent à rien, si les jeunes usagers ne se plient pas au savoir-vivre en bibliothèque, bref si tel ou telle persiste à confondre mutisme et silence ou expressivité juvénile et expression de soi, elles veillent et punissent :

[...] un peu d'agitation vers $6 \mathrm{~h}$; il faut en faire sortir plusieurs et le calme se rétablit ${ }^{26}$.

[...] les petits employés et ouvriers qui viennent généralement le samedi après-midi ont fait beaucoup de progrès depuis l'année dernière : il est vrai que les plus désagréables ont été expulsés ${ }^{27}$.

Les résistances au règlement sont nombreuses - et les exemples de conduites déviantes prennent des formes variées. Tout se passe le plus souvent comme si les titis étaient partagés entre la reconnaissance de la plus-value symbolique qu'apporte la fréquentation du lieu et la méconnaissance délibérée de sa logique culturelle propre. Le petit apprenti Bic par exemple vient se mettre au chaud à la bibliothèque et s'amuse à importuner ses camarades avec des pratiques dignes de la guerre des boutons :

Bic est venu tout l'après-midi. Il était dans un mauvais jour. Il lançait des caoutchoucs à ses camarades. Le marmiton (un jeune apprenti cuisinier) ne bronchait pas. Ai conseillé à Bic de remettre ses caoutchoucs dans la poche. Pendant que je m'occupais de son camarade, il a sorti de nouveau les caoutchoucs. Mlle Famin qui était assise à côté de lui, lui a dit : « Je crois que vous feriez mieux d'écouter Mademoiselle ! » Bic (tout petit, pantalon long, costume

24. Ibid., 3 décembre 1924, p. 8.

25. Dreyfus, Comptes rendus de la bibliothèque de Ménilmontant, 18 février 1932. 26. Huchet, Journal 1925-1926, samedi 14 novembre 1925, p. 1.

27. Ibid., 5 décembre 1925, p. 5. 
bleu d'apprenti) - «Oh ! Mademoiselle là-bas, elle est comme les autres, aucune femme ne me résiste ! » J'ai résisté cependant et sur mon conseil il est parti lancer ses caoutchoucs dans la rue ${ }^{28}$.

Le jeune garçon affiche un sexisme ordinaire, provocateur, mais qui s'avère peu efficace : n'importe qui ne peut affirmer n'importe quoi, ou seulement à ses risques et périls. L'énoncé performatif du petit Bic n'a aucun pouvoir : « la magie des mots ne peut opérer ${ }^{29} \gg$ parce que le locuteur n'a aucune légitimité reconnue par son interlocutrice qui l'a déjà désavoué dans la parenthèse descriptive où elle fait son portrait. Deux univers langagiers se font face mais dans la salle de lecture la verve machiste populaire n'a pas sa place. Le petit Bic ne le savait pas, il l'apprend à ses dépens.

La mixité pratiquée à la bibliothèque alimente aussi ces bruyantes manifestations d'opposition. L'apprentissage de la mixité ne se fait pas en effet sans difficulté. Dans les années 1920 et 1930, il s'agit d'une expérience nouvelle en rupture totale avec l'école : « Ca sent la fille ${ }^{30}$ » s'exclame le jeune Robert qui, en fronçant le nez, entre dans la salle de lecture de Ménilmontant. Claire Huchet notera avec perspicacité le propos d'une visiteuse par ailleurs « enthousiasmée » par la bibliothèque L'Heure joyeuse : « Une seule chose semble l'inquiéter, c'est la camaraderie entre filles et garçons ${ }^{31}$. » Ce n'est que bien plus tard, à partir de la fin des années 1950 que le gouvernement favorisera la généralisation de la mixité scolaire. Mais, comme le rapporte Marguerite Gruny « à cette époque (celle du début de L'Heure joyeuse), en dehors de leur famille, garçons et filles vivaient séparés; séparés dans les établissements scolaires, séparés dans les patronages du jeudi, séparés dans les mouvements scouts en plein essor ${ }^{32}$. » On comprend

28. Huchet, Journal 1924-1925, 16 décembre 1924, p. 13.

29. Bourdieu, 2001, p. 108.

30. Dreyfus, Comptes rendus de la bibliothèque de Ménilmontant, 11 novembre 1931.

31. Huchet, Journal 1926-1927, 15 juin, p. 77.

32. Lors de la conférence faite à La Joie par les livres en 1985 (cf. note 10), Marguerite Gruny précise à propos de la mixité à la bibliothèque : «Un autre attrait était la mixité, aimant si puissant chez les adolescents que quelques-uns d'entre eux trichèrent sur leur âge pour être admis. C'est qu'à cette époque, en dehors de leur famille, garçons et filles vivaient séparés; séparés dans les établissements scolaires, séparés dans les patronages du jeudi, séparés dans les mouvements scouts alors en plein essor. Aussi, avec quel plaisir ils se rencontraient à la bibliothèque! Les filles se montraient discrètes dans l'expression de leur satisfaction, mais les garçons souvent ne cachaient pas leur félicité ; combien nous l'ont confié depuis! « On venait à la bibliothèque plus pour parler avec des filles que pour 
alors l'attitude du jeune G. qui passe outre les menaces d'exclusion réitérées des bibliothécaires, tout attiré qu'il est par les petites lectrices :

G. (12 ans) est mis à la porte de la bibliothèque pour 8 jours; il s'entêtait à se promener continuellement dans la salle, principalement du côté des filles, il était prévenu depuis déjà longtemps ${ }^{33}$.

Les filles, elles-mêmes, ne sont pas insensibles à la présence des garçons. Par leurs conduites bruyantes, elles vont jusqu'à provoquer le chef [élu pour le mois] qui assure le bon fonctionnement de la salle. Et ce, au risque d'une possible mise à la porte :

Achille fait bien son service, mais les filles seraient insupportables, rien que pour le plaisir d'être grondées par Achille. Nous lui conseillons de venir nous avertir et de ne point faire les observations lui-même ${ }^{34}$.

Le respect mutuel et la collaboration entre les garçons et les filles sont le but visé. La communauté des lecteurs s'invente et se construit ses règles de socialisation. Elle doit aussi les mettre en œuvre au fur et à mesure et c'est difficile.

Aussi nos bibliothécaires font preuve d'une grande bienveillance éducative. Elles ont pris conscience au fil du temps de l'ampleur de la tâche. Dès mars 1925, dans la revue de La Nouvelle Éducation, Claire Huchet écrit : « Il ne faudrait pas s'imaginer que tout est parfait à L'Heure joyeuse : il y a des orages et des nuages,

lire ! » Mais l'époque était chaste, et si de tendres liens se nouèrent, il semble bien qu'ils restèrent toujours dans les limites de la très pure idylle. Toutefois la mixité n'alla pas sans inconvénients. D'abord, elle n'était pas toujours bien admise par les éducateurs (dont les parents), et si les filles furent moins nombreuses à s'inscrire, c'est que, plus tenues que les garçons, elles n'obtenaient pas toujours l'autorisation parentale réglementaire. On vit aussi de déplaisants petits drames de la jalousie, dus au manque d'habitude des jeunes de se trouver ensemble, et même parfois de violentes querelles à la porte de la bibliothèque. Aussi, quand la bibliothèque de la mairie du cinquième fut quelque peu modernisée, avons-nous profité de l'occasion pour abaisser à quinze ans l'âge limite de l'inscription. Les difficultés dues à la mixité ne furent pas forcément toutes résolues, mais elles n'eurent pas la même ampleur et nous absorbèrent moins. »

33. Huchet, Journal 1925-1926, 10 juillet 1926, p. 91.

34. Ibid., 5 mars 1926, p. 29. 
justement parce que nous vivons et que nous préférons la réalité imparfaite à l'apparence sans reproche ${ }^{35}$. »

Elles sont donc attentives aux oralités indisciplinées et ne cessent d'interroger cette fameuse 《 réalité imparfaite ». En présence de la même «bande agitée » qui arrive tous les soirs à 18 heures et qui bavarde, elles font le constat suivant :

Il est naturel qu'ils éprouvent ce besoin étant donné qu'ils sont contraints en classe depuis le matin ; mais c'est le système des études qu'il faut changer et non pas la bibliothèque ${ }^{36}$.

Le calme moins académique qu'elles attendent dans la salle est lui aussi réfléchi :

Il y a de l'agitation. Pas de dissipation mais le ronronnement de l'activité si familier à Croydon (il s'agit de la bibliothèque britannique dans laquelle a travaillé C. Huchet). Cependant combien de gens penseraient qu'il y a du désordre. Comme si l'ordre était l'immobilité. C'est évidemment plus difficile mais combien plus intéressant ${ }^{37}$.

L'implication active du lecteur est au cœur du dispositif qui fonctionne sur un mode relationnel et appropriatif inspiré de la Nouvelle Éducation. On est loin d'un mode de fonctionnement directif qui semble être celui auquel se réfère Madame Griffiths. Claire Huchet, encore elle, s'en rend parfaitement compte : « Madame Griffiths trouve les enfants bruyants; ce n'est pas notre avis ${ }^{38}$. » Aussi nos bibliothécaires ne renoncent-elles pas à se montrer fermes quand elles le jugent nécessaire :

À midi on lit avec attention, malheureusement quelques-uns sont en veine de farces; une odeur nauséabonde se répand dans la salle. Des boules ont été jetées. On soupçonne les coupables que nous faisons sortir sans être persuadées que ce sont eux les seuls coupables. Curieux gamins qui font des farces pour faire des farces, surtout aux camarades ${ }^{39}$.

35. Huchet, Journal 1924-1925, 1925, p. 28.

36. Ibid., $1^{\text {er }}$ décembre 1924, p. 7.

37. Ibid., 16 janvier 1925, p. 24.

38. Ibid., 22 octobre 1925, p. 119.

39. Ibid., 21 février 1925, p. 38. 
Les boules puantes relèvent moins des oralités comme pratique ordinaire, oralité spontanée du cri par exemple, mais plutôt des oralités comme culture, culture folklorique enfantine ici. Elles sont une réponse au conflit culturel qui se joue dans la salle entre ceux qui lisent avec attention et «les curieux gamins ». D'un côté les pratiquants de la culture écrite - « ce grand silence à l'intérieur duquel l'individu s'aménage une sphère privée et libre ${ }^{40} \gg$, de l'autre les tenants de la culture orale de la rue, collective et publique, irrespectueuse et joyeuse qui exhibe ce que les autres cachent : les odeurs d'excréments qui agressent les corps et exacerbent les sens « primaires ». En charivarisant le monde ordonné de la bibliothèque, ces corp/oralités contestataires remettent au premier plan « le bas corporel et matériel ${ }^{41} \gg$, que la lecture avait annihilé.

Les boules puantes, provocation scatologique gamine et populaire, ne sont pas admises, pas plus que les chansons qui gênent les autres et perturbent le calme requis :

Pauvre Daniel ! Il est mis à la porte parce qu'il chante malgré nos observations; sanglots. Il faut tout de même leur apprendre le respect des autres ${ }^{42}$.

Les sanglots sont révélateurs d'une véritable incompréhension culturelle qui produit de la violence symbolique. Le pauvre Daniel n'a pas les usages du lieu et il chante malgré lui, parce qu'il est heureux peut-être, un peu comme le fait l'ouvrier de la rue de Ménilmontant quand il fabrique une pièce, par exemple. Cette forme d'oralité de résistance demande, pour être dépassée, un changement de système culturel qui nécessite plus que quelques « observations ». Aussi le « respect des autres » doit-il être compris comme une adhésion aux valeurs légitimes du lieu, valeurs partagées par les autres, les lecteurs qui ne sont pas mis à la porte et auxquels le jeune Daniel est sommé de s'identifier.

Si faire communauté de lecteurs implique l'adhésion à des normes de conduites et à des valeurs communes, être chassé de la salle de lecture peut faire naître un fort ressentiment chez certains exclus et alimenter leur colère :

Nous faisons une remarque à Loulou qui colle des fiches de prêt : coup de tête, bougon, s'en va. Moreau ne vient pas. À la fin de la séance on entend des allers et venues dans l'escalier, on jette des

40. Furet \& Ozouf, 1977, p. 358.

41. BAKHTINe, 1970, p. 186.

42. Dreyfus, Comptes rendus de la bibliothèque de Ménilmontant, 16 janvier 1932. 
pierres et tout à la fin - S. Assaël est déjà partie - la porte est ouverte brusquement et nous lance un canif fermé avec un papier maintenu par une des lames : « Mademoiselle Jacqueline si vous rentrez chez vous, c'est la Mort signé la main noir recherché par la police depuis si ans par la rousse ${ }^{43}$. »

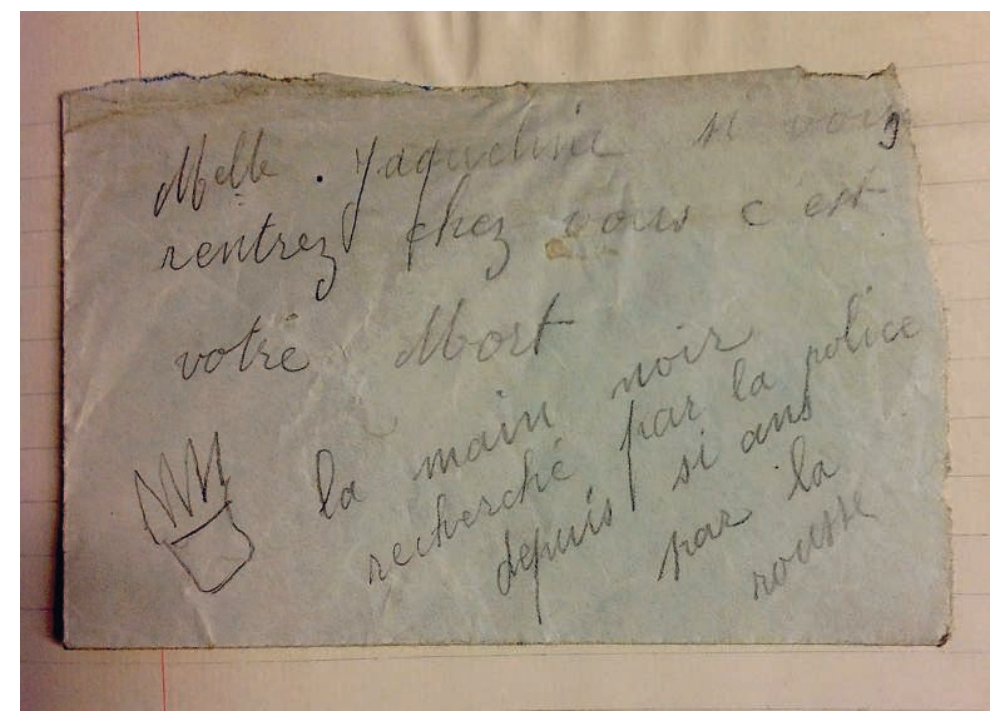

Illustration 3

« Le mot » Jacqueline Dreyfus, Comptes rendus de la bibliothèque de

Ménilmontant, 16 janvier 1932

Fonds patrimonial Heure joyeuse

Quelques mois plus tard le fameux Loulou est mis à la porte 8 jours, il traite la bibliothécaire « de son haut ». Et cette dernière de conclure : «Une fois pour toutes c'est dit je ne lui damnerai pas le pion ${ }^{44}$. » Devant les menaces réitérées $\mathrm{du}$ loulou en question, elle fait venir un agent mais il n'y a personne. Loulou ne viendra plus à la bibliothèque ! Échec de l'acculturation au monde d'une culture écrite policée, incorporée, valorisée. Loulou résiste comme un beau diable et fait feu de tout bois. Ces oralités de résistance se déclinent diversement aussi bien dans leur forme qu'en intensité. Le bougon qui murmure entre ses dents quitte

\section{Ibid.}

44. Ibid., 19 octobre et 26 novembre 1932. 
la salle sur un coup de tête dit la bibliothécaire. Peut-être qu'en dépit de ses efforts (il colle des fiches de prêt), il se sent humilié dans sa virilité de petit chef de bande naissant, d'être repris par une femme ? Les choses n'en restent pas là, le rejet actif et inventif des postures et des valeurs du lieu s'organise : allers et venues intempestifs, jets de pierre et autres façons de faire du dehors qui s'insinuent dans l'escalier. Enfin coincée dans un canif, la menace écrite dans une orthographe plus ou moins phonétique offre un bel exemple d'hybridation des modes de communication. La menace verbale lancée au visage de l'adversaire selon le code de l'honneur des petits voyous en herbe croise la littérature populaire et ses héros emblématiques comme Fantômas, le maitre du crime. Loulou appartient à la rue et à ses codes qu'il ne peut «ex-territorialiser » dans le monde ordonné de la culture écrite et de la bibliothèque. In fine il abandonne la place.

Tous les lecteurs mis à la porte ne réagissent pas aussi radicalement que Loulou mais les exclusions affectent ceux qui en sont frappés, tout au moins une partie d'entre eux :

Le soir on met un pétard à la porte ; sans doute un lecteur renvoyét5.

- Emmanuel, il faut veiller à ce qu'il n'y est rien d'écrit sur la porte. — Ah ! Mademoiselle, cela ne se renouvellera plus. J'sais qui avait écrit ça! Et si vous saviez ce que j'ai passé à ce camarades ${ }^{46}$.

Ces deux remarques parmi d'autres nombreuses renouent avec le paysage sonore des oralités de rébellion. Elles constituent une partie du quotidien de la vie de la bibliothèque où les bruits, les cris, les « engueulades » autour de la porte montrent peut-être chez certains lecteurs un début de crédit dans la légitimité du lieu.

\section{... forcer la porte ou tout au moins essayer}

Certains exclus voudraient bien revenir à la bibliothèque et être autorisés à passer la porte à nouveau. Mais le temps de l'exclusion doit être suffisamment long pour marquer les esprits et valoriser le moment où l'on sera autorisé à revenir :

45. Huchet, Journal 1925-1926, 3 février 1925, p. 22.

46. Ibid., 21 janvier 1926, p. 19. 
À la fermeture la bande agitée demande quand elle pourra rentrer ; il faut se faire violence pour ne pas dire demain ${ }^{47}$.

Il faut adhérer aux règles du groupe et accepter les sanctions qui les accompagnent quand la règle commune n'est pas acceptée. Quand on a été chef, c'est-à-dire garant du règlement et de son application, l'acceptation du renvoi est difficile : «L'ancien chef MF, vexé de ne pouvoir entrer, essaie de faire du bruit à la porte ${ }^{48}$. $\gg$ Deux jours avant, l'entrée de la bibliothèque lui a été interdite pendant trois semaines. Claire Huchet admet que c'est beaucoup mais précise aussitôt qu'il le mérite largement.

Le mot « bruit » dans l'expression « faire du bruit à la porte » est assez vague mais ici, dans le contexte donné, il s'agit très certainement de cris, de hurlements ou de jurons proférés depuis la frontière culturelle invisible que matérialise la porte. Agression sonore lancée à tue-tête, le bruit à la porte s'affiche comme l'envers des paroles mesurées des bibliothécaires. Cette protestation vulgaire est une tentative de rabaisser, de dévaloriser et même de démystifier la bibliothèque et son aura « sacrée » et solennelle de lieu des livres. Peut-être parce que l'on a commencé à y croire ?

D'autres jeunes lecteurs [ce sont surtout des garçons] renvoyés ravalent leurs cris, domestiquent leurs corps, renoncent à l'affrontement et s'essaient à négocier :

Schiess est venu demander s'il pouvait rentrer. Le « non » est tombé comme une douche. C'était pénible à faire mais nécessaire ${ }^{49}$.

Il revient à la charge un peu plus tard, le même jour, mais cette fois il change de stratégie :

Schiess vient faire des excuses pour rentrer mais nous demeurons inflexibles quoi qu' il nous en coûte ${ }^{50}$.

Les négociations symboliques et les calculs éducatifs sont multiples. Il y en a même qui recourent à des intermédiaires pour faire aboutir leur requête :

47. Huchet, Journal 1924-1925, 13 décembre 1924, p. 13.

48. Ibid., 10 juillet 1925, p. 91.

49. Huchet, Journal 1925-1926, 5 février, p 22.

50. Ibid. 
Vandergracht fait demander par ses camarades quand il pourra rentrer. Il fait cela depuis plusieurs jours. « Eh bien Vandergracht en partant l'autre jour vous aviez dit qu'on ne vous verrait plus à L'Heure joyeuse. » - « Oui mais je croyais que vous étiez beaucoup plus fâchée que vous l'êtes et comme vous n'êtes pas méchante ... » «Ah! Je vois vous voulez me prendre par les compliments. » Rires des camarades. « Non, j'fais pas de compliments. J'aime pas ça ; c'est pas pour ça ${ }^{51}$.

Les lecteurs doivent comprendre que le bon fonctionnement de la bibliothèque dépend d'eux. Il faut donc développer leur sens de la responsabilité et « éveiller leur esprit d'initiative » :

La petite fille renvoyée pour 15 jours vient rapporter ses livres et faire une petite scène. Cependant après une explication elle reconnaît que nous avons raison et s'en va en promettant de revenir ${ }^{52}$.

\section{... franchir à nouveau la porte}

Être autorisé à passer à nouveau le seuil est un moment fort. Et le retour doit s'accompagner d'un véritable changement de conduite à l'instar de Schiess, élu chef alors que l'année précédente il a été « tant de fois à la porte ». Aussi d'emblée certains lecteurs donnent des gages du cheminement parcouru pendant leur exclusion et des bonnes dispositions dans lesquelles ils se trouvent :

La bande Joly revient un peu calmée. «C'que ça m’a manqué, Mademoiselle, de n'pas v'nir à la bibliothèque ! Ah ! je suis bien guérie de parler $^{53} !$ »

Mais l'incorporation des façons de faire et des façons de dire de la salle de lecture n'est pas aisée :

Grisez et Guéno sont revenus. Ils ont encore du mal à rester tranquille; quand ils sont seuls, c'est parfait ${ }^{54}$.

51. Huchet, Journal 1924-1925, 19 octobre, p. 118.

52. Huchet, Journal 1928, 28 février 1928.

53. Huchet, Journal 1925-1926, 14 janvier 1926, p. 16.

54. Ibid., 30 janvier 1926, p. 21. 
Il arrive même que des lecteurs renvoyés alternent retours et exclusions et ce malgré des excuses où ils reconnaissent avoir été « insupportables ». C'est alors le retour de ces oralités contestataires où s'imposent la langue de la rue et la libre et bruyante expressivité des corps.

Cependant, quand, de nouveau on les reprend « à l'essai », ils recommencent les mêmes bavardages et les mêmes grossièretés ${ }^{55}$.

Ces recommencements incessants, « ces adhésions à éclipses » (Hoggart, 1970, p. 327-333) sont le signe des tensions extrêmes éprouvées par certains lecteurs qui n'arrivent pas à faire le deuil de l'autre monde culturel, celui de la rue par exemple où les grossièretés clamées à haute voix sont parfaitement admises. Dans la bibliothèque, ces grossièretés sont volontairement proférées comme une violation flagrante des règles de civilité admises, comme un refus délibéré de se plier aux conventions verbales du lieu : la politesse, le respect de la bienséance et des convenances. Mais le retour régulier des coupables contrits montre là aussi que le long travail d'acculturation est en marche, que « le passage d'une culture à l'autre est un processus lent, partiel comme une interminable déchirure ${ }^{56} \gg$.

Alors le groupe des lecteurs - de ceux qui ne font pas changer « l'atmosphère de la salle » en étant grossiers - cherche à se faire une idée de la réalité de la transformation opérée et manifeste peut-être un léger doute :

Aujourd'hui, rentrée de ceux qui ont été mis à la porte. Ils sont assez sages. Les autres les regardent avec curiosité ${ }^{57}$.

Ce qu'attendent avec intérêt nos bibliothécaires, ce sont des régulations entre pairs qui coupent court à toute résistance :

Un garçon ferme son livre bruyamment. Toute la table le gronde, les autres se retournent, mécontents $-\ll$ T'es pas malade ${ }^{58}$.

Mais ces régulations doivent être comprises et acceptées par tous :

Bruit à la porte. Kroeger va chercher un agent. Nous en profitons pour faire comprendre que le chef n'est pas un surveillant mais un ami [...] Schiess (il vient d'être élu chef et a un passé de forte tête)

55. Gruny, Leriche, Journal 1930, 18 mars 1930.

56. Furet \& Ozouf, 1977, p. 368.

57. Huchet, Journal 1924-1925, 16 décembre 1924, p. 13.

58. Ibid., 13 décembre 1924, p. 12. 
dit qu'il a compris et avec un grand effort ajoute qu'il ira chercher un agent s'il est nécessaire. La vie sociale de la bibliothèque prend forme ${ }^{59}$.

Le but ultime recherché est la « self discipline » :

Nous faisons rentrer les espiègles d'hier en leur expliquant le système de self discipline sur lequel fonctionne la bibliothèque. Ils sont fort penauds et sont comme des souris toute l'après-midi ${ }^{60}$.

Voilà un bel exemple parmi d'autres de compromis culturel où la self discipline est explicitement pratiquée : «J'viendrai pas c'après-midi parce que j'sens que ça n'serait que pour chahuter ${ }^{61}$. » Le parler juvénile populaire reproduit ici par la bibliothécaire attire l'attention sur les traits articulatoires et donc sur l'habitus linguistique (dispositions socialement façonnées) du jeune locuteur. Certes Claire Huchet montre ainsi le décalage avec son propre habitus linguistique et le parler attendu à la bibliothèque. Mais, dans le même mouvement, elle montre aussi que

[...] la contrainte que le marché exerce par l'intermédiaire de l'anticipation des chances de profit prend naturellement la forme d'une censure anticipée, d'une autocensure, qui détermine non seulement la manière de dire, c'est-à-dire le choix du langage $[\ldots]$ mais aussi ce qui pourra ou ne pourra pas être $\operatorname{dit}^{62}$.

Et nous ajouterions volontiers : ce qui pourra ou ne pourra pas être fait. L'aveu que fait le jeune garçon s'exprime sans timidité ni véritable insécurité et presque avec une certaine assurance. Cela tient certainement à la relation établie avec la bibliothécaire et à son attitude encourageante qui permet d'anticiper des profits positifs. Les oralités de résistances n'ont alors plus vraiment de raison d'être. Inutiles en salle de lecture, elles retournent dans la rue, d'où elles viennent d'ailleurs. Elles cèdent la place aux oralités joyeuses comme celles du jeune Schwartz :

59. Ibid., 8 octobre 1925, p. 114.

60. Ibid., 27 novembre 1924, p. 6.

61. Ibid., 10 octobre, p. 114.

62. Bourdieu, 2001, p. 115. 
Il adore la bibliothèque et Mlle L. et il blâme beaucoup plusieurs de ses camarades qui lui avaient dit de venir pour « chahuter » parce qu'il n'y avait que trois jeunes filles. « Eh bien et votre chahut ? » - «Oh Non! Quand on a vu comment c'était ! » Et il parle avec tendresse de la bibliothèque. Il est très touché d'être traité en homme, fier de la confiance des bibliothécaires et voudrait que tout le monde comprenne que c'est rare ${ }^{63}$.

\section{L'Heure du conte et ses auditeurs}

Nos bibliothécaires sont particulièrement attachées à l'Heure du conte qui, dans le dispositif général de la bibliothèque, est un temps fort de l'appropriation du livre. « Nous devons certainement faire porter notre effort sur l'Heure du conte ${ }^{64}$ » note ainsi Jacqueline Dreyfus en 1932. Si, dans la salle principale, la lecture de livres est prise dans un réseau de sociabilités liseuses multiples et implicites qui en atténue la dimension solitaire, l'Heure du conte déploie des stratégies d'esquive de l'écrit ${ }^{65}$. Ces stratégies qui passent par l'oralisation des histoires (lecture à haute voix, contage) et par la constitution d'un groupe d'auditeurs partageant des expériences et des émotions ${ }^{66}$ sont conçues comme facilitatrices. Mais la présence d'une conteuse, diseuse de textes et passeuse de lecture, n'empêche pas l'expression d'oppositions culturelles et de véritables oralités de résistance de la part du jeune public.

\section{Oralités belliqueuses : les retardataires}

Pour assister à cette fameuse Heure du conte et faire partie du groupe des auditeurs, il faut arriver à l'heure, précisément. Une fois la porte fermée, il n'est plus question d'entrer. Ce moment particulier de partage d'une histoire, cette « communion bienfaisante » selon l'expression de Mathilde Leriche, doit être protégée des turbulences extérieures. Mais pour cela, encore faut-il comprendre l'intérêt de la règle, se sentir responsable du bon fonctionnement du lieu. Cette « éducation » culturelle rencontre donc parfois de grandes résistances.

63. Huchet, Journal 1925-1926, 13 janvier 1926, p. 15.

64. Dreyfus, Comptes rendus de la bibliothèque de Ménilmontant, 3 février 1932.

65. NAFFrÉCHOUX, 1987, p. 404.

66. Ibid., p. 409. 
Voici une petite chronique de ces oralités belliqueuses dans les années 1928-1929, au tout début de la bibliothèque de Ménilmontant :

[...] quatre ou cinq enfants expulsés, non-inscrits à la bibliothèque, mais venus lire et écouter le conte, manifestèrent bruyamment une fois dehors. Quelques lecteurs arrivés en retard et non admis au conte se joignirent à eux : coups de poing et de pieds dans la porte, pierres lancées, injures.

À la fin du conte, les émeutiers firent une brusque irruption dans la Bibliothèque jetant des livres et le contenu des fichiers de cartes postales par terre. La bibliothécaire était restée seule; elle ne pouvait donc pas abandonner les lieux et chercher l'aide du gardien de la cité ou des agents. N'obtenant aucun résultat par ses paroles, moins forte physiquement que ces enfants, elle attendit leur départ...

Les émeutiers vinrent troubler les cinq ou six séances de prêt suivantes et brisèrent même un carreau. L'une des bibliothécaires se vit alors contrainte de garder la porte, ne l'ouvrant qu'aux têtes connues. Nous avertîmes les agents pour les faire appeler en cas de besoin. Mais peu à peu le calme se fit et malgré quelques récidives, les « émeutiers » vinrent même lire sur place et, très sages écoutèrent un conte ${ }^{67}$.

Si nos bibliothécaires ne baissent pas les bras et parviennent à faire exister l'Heure du conte dans cette cité d'habitations à bon marché, l'appropriation culturelle est présentée comme un violent combat (le terme émeutiers revient par trois fois) et la bibliothèque se transforme en une véritable citadelle assiégée. Mise à sac, recours à la force (les paroles sont vaines), bris de glace, filtrage à l'entrée, protection assurée par des agents de police : la violence physique de cet affrontement est une réponse à la violence symbolique imposée (tout au moins vécue comme telle) par la présence d'un lieu de culture cultivée et de ses règles de fonctionnement. Les récidives comme les moments où « très sage » on écoute un conte sont autant de signes de réappropriations difficiles à négocier.

Moins agressifs mais tout aussi perturbateurs, certains retardataires dont les arrivées intempestives mettent en péril l'ambiance indispensable au partage des

67. Dreyfus, Office des habitations à bon marché. 140, rue de Ménilmontant, Paris, $20^{\mathrm{e}}$ arrondissement. Bibliothèque de la Garderie. Rapport de fin d'année, 1928-1929, 1930-1931. 
histoires, profitent des failles du dispositif, uniquement préoccupés par leur bon plaisir :

[...] un peu de désordre parce que la chaîne n'est pas fermée en bas de l'escalier et deux ou trois lecteurs entrent pendant l'histoire ; assis au loin ils entendent mal et relâchent le « cercle ${ }^{68}$.

ou encore :

D'autres arrivent à la dernière minute et même pendant l'histoire et une fois la chaîne fermée ${ }^{69}$.

Le sentiment de responsabilité par rapport au groupe ne peut que se développer petit à petit, en acquérant des habitudes et des aptitudes :

Nous apprenons, en sortant, qu'un petit groupe d'habitués est arrivé en retard. Il avait, paraît-il, l'air navré et l'un d'eux s'est mis à pleurer à chaudes larmes ${ }^{70}$.

\section{Oralités bruyantes : les entrées et les sorties, les allées et venues, les mal assis}

Alors que les « émeutiers retardataires » sont relativement rares, il y a, par contre, des moments particulièrement propices à la manifestation d'oralités indisciplinées qui viennent parasiter la démarche de constitution d'un auditoire. Aussi nos bibliothécaires sont-elles très attentives aux entrées et aux sorties des Heures du conte et à l'installation du public : une bonne partie de la réussite du contage ou de la lecture se joue dans ces espaces préparatoires. En effet, selon elles, « les heures du conte ne sont pas ce qu'elles devraient être [si] les enfants n'oublient pas toute autre chose pendant qu'ils écoutent l'histoire ${ }^{71} \gg$. Les notes sur les fiches qui rendent comptent des séances de contage signalent à plusieurs reprises les problèmes posés par ces débuts et de ces sorties :

Du mal au début pour que tous soient tranquilles ; plusieurs petits un peu agités. Nous recommençons le début de l'histoire ${ }^{72}$.

68. Dreyfus, Heure du conte, 10-12 ans, 7 mars 1932.

69. Ibid., Petits 9 ans, 19 décembre 1936-1937.

70. Gruny, Heure du conte, 9-10 ans, 2 juillet 1931.

71. Dreyfus, Heure du conte, 20 décembre 1934.

72. Leriche, Heure du conte, 10 ans, 13 avril 1933. 
Installation et départ bruyant : le samedi les enfants viennent au conte en sortant de l'étude et aujourd'hui Cuny est de la partie qui chante l'Internationale à tue-tête dans le vestiaire [où se déroule l'Heure du conte] ${ }^{73}$.

Galopade à la fin comment l'éviter ${ }^{74}$.

Toujours sortie très houleuse et décourageante ${ }^{75}$.

Une installation chaotique, une lecture mal maîtrisée, un public hétérogène, ouvrent des brèches aux échappées dissipées : brouhaha, cris, manque d'attention.

Essai d'Heure du conte dans la cour mais on est dérangé ; bruit, va-et-vient et à la fin les orphelines en horde sauvage et hurlante $[\ldots]^{76}$.

[...] nous nous sommes laissée entrainer à lire longtemps d'où chaises qui se balançaient, pieds qui remuaient, conversations, batailles même, à tout ceci se mêlent des « astuces », des exclamations d'approbation ou de critique ; enfin l'atmosphère est assez troublée et sans rien de cette douceur apaisante et harmonieuse qui doit être celle de l'Heure du conte ${ }^{77}$.

C'est la première fois que nous racontons dans le vestiaire, aussi nous sommes très mal installés, les enfants sont serrés, il fait chaud, nous n'en voyons pas la moitié ; enfin le public n'est pas formé $\mathrm{du}$ tout (beaucoup de lecteurs viennent écouter les histoires pour la première fois), l'installation prend une demie heure. Ce sont là autant de conditions défavorables auxquelles il faudra remédier ${ }^{78}$.

73. Dreyfus, Heure du conte, Petits 9 ans, 20 novembre 1936-1937.

74. Ibid., 9-11 ans, 3 janvier 1935.

75. Ibid., Petits 9 ans, 12 décembre 1936.

76. Leriche, Heure du conte, 10 ans, 19 mai 1932.

77. Dreyfus, Heure du conte, 16, 17, 18 avril 1935-1936.

78. Gruny, Heure du conte, 8 à 15 ans, 25 novembre 1938. 
CAHIERS DE LITTÉRATURE ORALE

128 L'Heure du conte $-n^{\circ} 86$

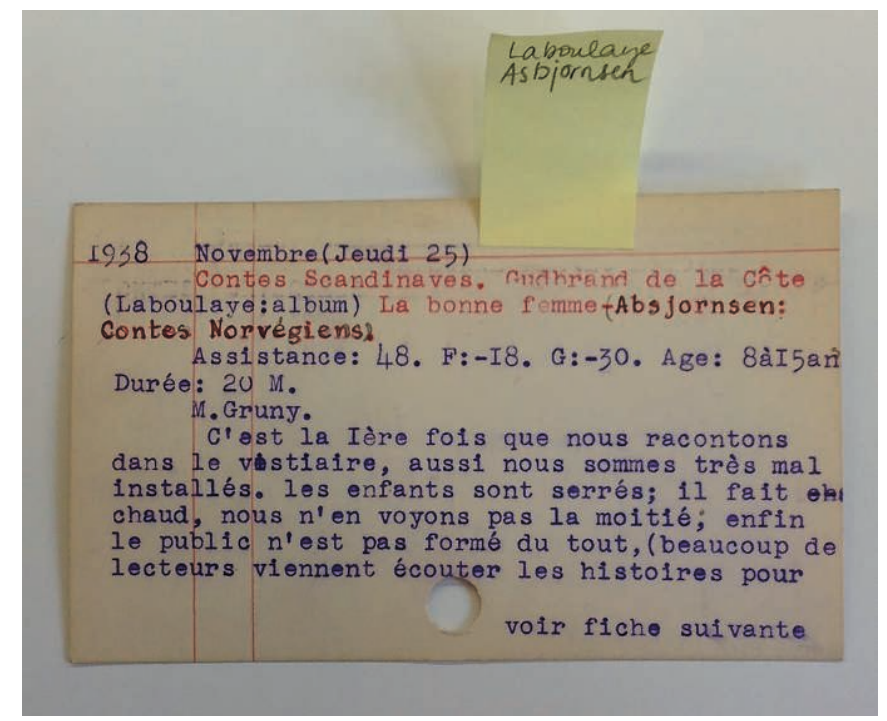

Illustration 4

Marguerite Gruny, Heure du conte, 25 novembre 1938 (1) Fonds patrimonial Heure joyeuse

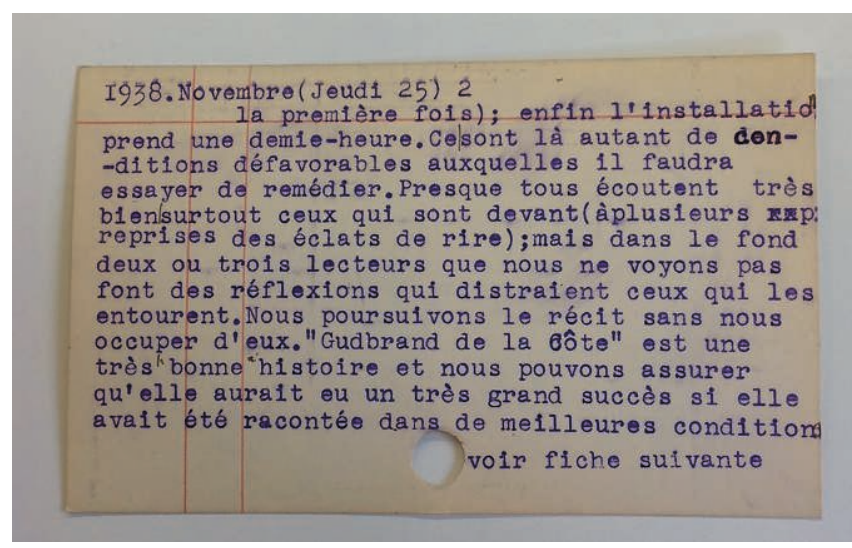

Illustration 5

Marguerite Gruny, Heure du conte, 25 novembre 1938 (2)

Fonds patrimonial Heure joyeuse 
Les enfants ont du mal à se glisser, de leur plein gré, dans la culture écrite après une journée de classe ou d'atelier (temps libre $v$ s temps obligé) et semblent toujours sur le point de s'évader :

Sont intéressés, parfois émus mais dans l'ensemble peut-être pas assez avancés pour jouir pleinement de tous les détails [...] Tous aiment l'histoire et veulent savoir la suite et pourtant attention difficile à maintenir, qui fléchirait facilement. Pourquoi ? On ne sait pas ; peut-être à cause de plusieurs nouveaux ${ }^{79}$.

\section{Oralités frondeuses : les petits groupes qui font sécession}

Quand les enfants sont enfin réunis et que l'Heure du conte a commencé, certains, par petits groupes, entrent en dissidence. Ils empêchent plus ou moins le public rassemblé de se transformer en un auditoire pris dans le discours de la conteuse. Il y a comme un refus de se laisser embarquer dans le cours d'une fiction dont on ne sait où elle pourrait conduire.

Plusieurs font des réflexions à tout propos, ce qui gêne les autres et la conteuse ${ }^{80}$.

[...] dans le fond deux ou trois lecteurs que nous ne voyons pas font des réflexions qui distraient ceux qui les entourent. Nous poursuivons le récit sans nous occuper d'eux ${ }^{81}$.

Ces oralités d'opposition frondeuses sont aussi des prises de position de jeunes auditeurs en formation qui ne se sentent pas en phase avec le récit proposé et qui demandent, peut-être, à travers ces protestations, un meilleur ajustement à leur goût. Une amorce de compromis culturel ?

Le conte semble émouvoir vivement la plupart des enfants. Malheureusement nous sommes très mal installés. Nous ne voyons pas tous les auditeurs, aussi une bande de quatre ou cinq petites filles de 10-12 ans pourtant habituellement gentilles, font les petites sottes aux passages les plus sentimentaux, ce qui trouble l'atmosphère. À partir de la prochaine fois, puisqu'il n'est

79. Leriche, Heure du conte, 10 ans, 16 février 1933.

80. Halff, Heure du conte, 12 février 1931.

81. Gruny, Heure du conte, 8-15 ans, 25 novembre 1938. 
pas possible de voir ainsi les auditeurs placés au fond de la salle, nous nous assoirons sur une petite table ${ }^{82}$.

\section{Oralités rétives : l'indocile, l'esprit fort, le mime, la « bricoleuse »}

Il y a aussi dans l'assistance, alors que la conteuse a commencé son récit et cherche à prendre les enfants dans le « charme » de sa parole, des individus isolés qui font cavalier seul :

Arnoux n'écoute pas bien. Il a passé son temps à se mouiller les cheveux et à donner des coups de poings à son voisin ${ }^{83}$.

Les enfants écoutent dans le plus grand silence [...] excepté Ludurkovosky qui n'est nullement pris et fait des grimaces ${ }^{84}$.

Si certains se refusent à suivre le mouvement général par jeu provocateur avec l'adulte, le ricanement est pour quelques adolescents (16 ans dans l'exemple ci-dessous) une manière d'afficher une opinion personnelle qui signale, peut-être, un esprit en formation. Ainsi à la lecture de La petite communiante de Félix Timmermans, Claire Huchet note :

[...] Un seul ricane et encore n'est-ce là sans doute que pour faire l'esprit fort. Impression que ceux qui étaient gênés auraient apprécié si l'autre sexe n'avait pas été là ${ }^{85}$.

Mais les ricanements peuvent être la marque d'un décalage entre l'auditeur et le texte offert. Ces jeunes adolescents - l'un d'eux surtout - ne veulent pas s'en laisser conter :

La tournure poétique de l'histoire les surprend; les détails gracieux leur semblent puérils. On aurait dû les prévenir que cette histoire était un conte. Plusieurs garçons ricanent et apportent un esprit laid, un surtout. Atmosphère gênante pour la personne qui

82. Gruny, Heure du conte, 8-15 ans, $1^{\text {er }}$ décembre 1938.

83. Dreyfus, Heure du conte, 9-12 ans, 28 février 1935.

84. Ibid., 23 mai 1934-1935.

85. Huchet, Heure du conte, âge moyen 16 ans, 28 avril 1928. 
raconte. Plusieurs néanmoins écoutent avec grand plaisir et sont pris par l'histoire ${ }^{86}$.

Mathilde Leriche a lu La Légende de Flore et Blanchefleur, un poème du $\mathrm{XII}^{\mathrm{e}}$ siècle. Elle note après sa prestation : «On aurait dû les prévenir que cette histoire était un conte. » Elle donne également son point de vue en signalant « un esprit qui est laid. » Toute proportion gardée, la position de notre conteuse rappelle celle du poète médiéval Philippe de Rémi dans son prologue à la Manekine :

[...] Mais s'il y a dans l'assistance quelqu'un qu'afflige l'idée d'entendre une histoire morale, au nom de Dieu, qu'il s'en aille sans tarder ! Car ce n'est faire preuve ni de courtoisie, ni de bon sens que de troubler le conteur. [...] Je veux leur demander de s'en aller, ou bien de n'élever ni querelle ni contestation. Car, à quoi sert une belle histoire, si elle n'est pas reçue du fond du cœur, et tout particulièrement par ceux qui l'entendent raconter ${ }^{87}$ ?

Face à un jeune public en formation, la conteuse a pris soin de choisir la version « renouvelée de J. Marchand ». Et pourtant il y a maldonne pour au moins un membre de l'assistance. Ce dernier se sent déconsidéré par l'adulte qui semble le ramener à l'enfance toute proche par un texte aux « détails puérils ». Il le fait brutalement savoir et formule sûrement des remarques à caractère sexuel pour montrer qu'il a grandi (et qu'il est un vrai garçon...) ! Il y a des enfants qui apprécient l'histoire racontée mais participent à leur manière. Les uns en familiarité avec un récit déjà connu et fort bien mémorisé se plaisent à doubler la parole de la conteuse et sans doute à trouver grand plaisir à faire partie du petit cercle des initiés :

On est gêné par un lecteur qui connaissant l'histoire dit à mi-voix la suite avant nous. Plusieurs connaissent aussi mais ne disent $\operatorname{mot}^{88}$.

86. Leriche, Heure du conte, âge moyen 13-14 ans, 7 mars 1931.

87. Beaumanoir, 1995, p. 19.

88. Leriche, Heure du conte, 10 ans, 24 octobre 1929. 
[...] Freyer et Rosenblum, tout près de nous, content à voix basse l'histoire connue ${ }^{89}$.

Ce plaisir de re-connaissance conforte leur affiliation au groupe des fidèles de l'Heure du conte. D'autres peuvent participer par corps à la fiction qu'il leur arrive de mimer sur un mode enfantin en quelque façon, doublage qui perturbe évidemment l'écoute sereine et pleine de l'histoire :

Ce conte amusant plait à l'ensemble de l'auditoire mais Cuny est là au $1^{\text {er }}$ rang ! Il suit d'ailleurs bien mais le témoigne en mimant tout ce conte; chevauchant quand Mathieu saute sur son cheval, faisant la guerre comme il la fait; les autres voudraient qu'il se taise; son voisin et lui s'envoient des taloches; l'harmonie est très troublée, pas la paix souhaitée. Nous ne pourrons plus l'accepter [...] Des séances de ce genre sont néfastes et entament l'avenir ${ }^{90}$.

Ces formes d'écoute sont éminemment participatives, comme si l'esthétique de la réception devait s'accompagner d'une présence par corps au conte, une esthésie de l'écoute en quelque façon. Cuny, lui, pratique l'identification sur le mode immersif et turbulent. Il semble ne vouloir à aucun prix renoncer à cet amusement. Et ce corps présent, trop présent, n'est guère toléré par ses camarades ni par la conteuse.

Il y a enfin ceux qui occupent leurs mains... pour mieux écouter peut-être.

[...] Rachel joue avec un brin de laine. Raymonde feuillette son livre et après le conte nous le lui reprenons : inutile qu'elle l'emporte puisqu'elle l'a lu pendant le conte !!! À part cela silence religieux et intéressé 91 .

Ces activités annexes ne sont pas, elles non plus, du goût de la conteuse ! Elle pense qu'elles sont le signe d'un désintérêt, d'une mauvaise écoute. Le bon auditeur, c'est l'auditeur pris : les émotions se lisent sur son visage mais son corps captif est pratiquement immobile.

Peut-être Rachel et Raymonde renouent-elles, d'une certaine façon, avec les pratiques des veillées d'autrefois et les tâches routinières qui s'y pratiquaient.

89. Dreyfus, Heure du conte, 21 février 1935.

90. Ibid., 12 décembre 1935.

91. Ibid., des petits et des grands de 13, 15 ans, 10 janvier 1935. 
Dans un texte intitulé Le conteur, Walter Benjamin observe que « [...] c'est dans l'ennui que l'esprit se relâche le plus complètement ». Il précise quelques lignes plus loin qu'à l'époque moderne « il n'est plus d'activités qui soient intimement liées à l'ennui ». Or, ajoute-t-il, l'art de raconter les histoires se perd précisément « parce qu'on ne file plus et qu'on ne tisse plus en écoutant. » Et de conclure : «Plus l'auditeur s'oublie lui-même, plus les mots qu'il entend s'inscrivent profondément en lui ${ }^{92}$. » Il n'est pas sûr que le brin de laine de la jeune Rachel soit un obstacle à une écoute profonde et intéressée. Il peut même être une aide...

\section{Des oralités joyeuses au silence de l'auditoire conquis}

Il existe des manifestations d'oralité qui montrent que certains enfants se sont mis au diapason et sont entrés en parfaite harmonie avec la conteuse et son dispositif. Mathilde Leriche définit ainsi la relation qui se tisse entre le jeune public et la diseuse d'histoires :

Un lien d'affection, de confiance, de reconnaissance unit les enfants à l'adulte dispensateur de ce bonheur [celui des plaisirs de l'Heure du conte]. Lien délicat et précieux, amitié confiante qui permet de mieux comprendre et aider chaque enfant ${ }^{93}$.

On retrouve ce lien délicat et précieux, cette amitié confiante dans plusieurs séances d'Heure du conte. Les enfants tombent sous le charme de la conteuse magicienne des contes de fées et lui manifestent leur admiration sincère :

À la sortie [de l'Heure du conte] Claude Haas dit : « Il faudrait bien un bâton magique pour faire la police dans l'escalier $\gg^{94}$.

Au moment où l'on allume la lampe unique. Claude Haas « Mademoiselle, vous allez jeter un sortilège ?» (cf. Le chat qui s'en va tout seul qui leur a été lu il y a quelques temps) ${ }^{95}$.

92. Walter, 200, p. 126.

93. Leriche, 1956, p. 12-13.

94. Dreyfus, Heure du conte, âge moyen 10-12 ans, 4 avril 1935.

95.Ibid., 9-10 ans, 22 novembre 1935. 
[...] une tante nous raconte les réflexions de son neveu, auditeur des contes pendant «la saison dernière».

« Ah Mlle, vous pouvez dire que vous avez un admirateur ! » Il disait à mon mari «Ah je voudrais que tu l'entendes; elle raconte de ces contes la demoiselle ! Je ne sais pas si elle les invente mais $!^{96} \gg$

Ce conte plait encore plus que le premier. Des exclamations de satisfaction : «ça c'est une belle histoire ${ }^{97}$.

La confiance que le jeune public témoigne à la conteuse, se manifeste aussi dans l'abandon émotif qu'il donne à voir à l'adulte sans réserve aucune. Un très bel exemple nous est offert par Pinocchio que raconte Mathilde Leriche :

Succès grandissant. Cette histoire leur convient merveilleusement. Public qui réagit beaucoup. Vibre à toutes les aventures : il y a des silences lourds et des explosions de colère ou de désespoir. Aux passages tragiques 2 ou 3 petits se bouchent les oreilles. Les autres sont plus braves et accompagnent généreusement Pinokio $^{98}$ !!!

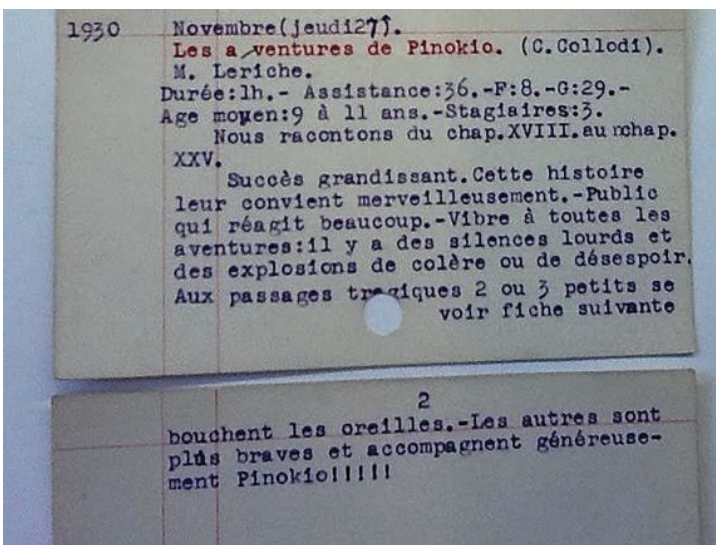

Illustration 6

Mathilde Leriche, Heure du conte, 20, 27 novembre 1930

Fonds patrimonial Heure joyeuse

96. Dreyfus, Heure du conte, 11 ans, 30 janvier 1936. 97. Leriche, Heure du conte, 10 ans, 27 avril 1933.

98. Ibid., 9-11 ans, 27 novembre 1930. 
Mais c'est le silence total qui est le signe incontestable de la réussite d'une Heure du conte. Nos bibliothécaires sont unanimes là-dessus et c'est ce qu'elles attendent avec un grand intérêt. Certains enfants qui ont déjà adopté la posture du parfait auditeur, veillent « au grain » :

[...] Plusieurs enfants comprennent le symbole et s'en réjouissent. [...] il y a des cris de protestations surtout parmi les garçons. Une ou deux filles qui comprennent bien leur imposent silence : «Tais-toi, tu ne comprends pas $!{ }^{99}$

Pierre Loreau se taille un succès par des remarques sans presque aucun rapport avec l'histoire, mais peu à peu les autres « pris » de plus en plus le font taire ${ }^{100}$.

Ce qu'il y a enfin d'obtenu est que cette fois enfin la discipline est intérieure. Quelqu'un demande qu'est-ce que c'est des « gonds ». Mais les autres le font taire d'une façon efficace et péremptoire $^{101}$.

Quand l'intériorisation des affects est maitrisée, le corps immobile et le silence palpable, l'auditoire est enfin constitué. Les expressions utilisées dans les fiches pour traduire ce moment privilégié sont toujours un peu les mêmes :

[...] Ils écoutent gravement la fin du récit ${ }^{102}$.

Silence recueilli des auditeurs pendant toute la séance $[\ldots]^{103}$.

Le conte et écouté avec un grand recueillement ; c'est la première fois de la saison que nous racontons un seul conte et le public est prêt ${ }^{104}$ !

99. Huchet, Heure du conte, 10 ans, 5 avril 1928.

100. Dreyfus, Heure du conte, 81/2-9 ans, 13 février 1936-1937.

101. Ibid., 24 janvier 1935.

102. Dreyfus, Heure du conte, 11-12 ans, 25 avril 1935.

103. Ibid., 16 janvier 1936.

104. Dreyfus, Heure du conte, 9 ans, 26 novembre 1936-1937. 
Après quelques minutes d'agitation au début, ils sont pris par l'histoire et écoutent avec attention ${ }^{105}$.

Très bonne séance ; attention profonde et sensible. La fin si belle les émeut beaucoup; ils restent silencieux un moment comme suspendus ; partis dans un autre monde ${ }^{106}$.

Certaines séances sont particulièrement remarquables à cet égard et montrent l'aboutissement de la formation d'un public en auditoire qui frôle la perfection :

Le succès de l'histoire est très grand ; on n'entend pas un souffle ; les auditeurs non seulement sont passionnés par le récit mais encore en apprécient le style moyenâgeux. Pendant toute la semaine ils nous poursuivent pour connaître la fin ${ }^{107}$.

Pour la première fois c'est ça [...] l'atmosphère, les enfants. Tout est incomparable. Un plein acquiescement général. C'est indéfinissable, «c'est ça ». Les enfants sont délivrés d'eux-mêmes, des contingences, de la minute présente, de l'installation, etc. ${ }^{108}$

Cet auditoire formé sait apprécier la fiction comme la narration. Il est aussi capable de s'absorber tout entier dans l'histoire racontée au point de s'affranchir de tout ce qui l'entoure. Il a acquis en quelque sorte les compétences nécessaires à la lecture personnelle qu'il va pouvoir pratiquer avec plus d'efficacité et d'intérêt en salle de lecture comme à la maison en empruntant des livres. La boucle est bouclée : les oralités matées ou canalisées, la lecture de la littérature, l'écrit retrouvent leur place à la bibliothèque.

Mais ce que nous montrent les documents que nous avons analysés, c'est que rien n'est définitivement gagné. Le trajet culturel accompli par les auditeurs-lecteurs qui vont de leur monde à celui de la bibliothèque est un passage sans arrêt renégocié pour un grand nombre d'entre eux. Chacun s'essayant à réduire l'interminable déchirure qui n'en finit pas de s'entrouvrir.

105. Halff, Heure du conte, 15 mars 1931.

106. Leriche, Heure du conte, 10 ans, 30 mars 1933.

107. Gruny, Heure du conte, 13 ans et demi, 14 octobre 1926.

108. Dreyfus, Heure du conte, 24 janvier 1935. 


\section{Bibliographie}

Bachelard Gaston, 1994 [1957], La Poétique de l'espace, Presses universitaires de France (coll. Quadrige), Paris, 215 p.

BakHtine Mikhaill, 1970, L'oeuvre de François Rabelais et culture populaire au Moyen Age et sous le Renaissance, Gallimard (coll. Bibliothèque des Idées), Paris, 473 p.

Beaumanoir Philippe (de), 1995, La Manekine, roman du XIII siècle, traduit et présenté par Christiane Marcello-Nizia, Préface de Donatien Laurent, Stock (coll. Moyen Age), Paris, 220 p.

Benjamin Walter, 2000 [1936], « Le conteur » in CEuvres III, Gallimard (Folio Essais), Paris, p. 114-151.

Bourdieu Pierre, 1980, Le Sens pratique, Les Éditions de Minuit (le sens commun), Paris, $374 \mathrm{p}$.

Bourdieu Pierre, 2001 [1980], « La formation des prix et l'anticipation des profits » in Langage et pouvoir symbolique, Éditions Fayard (coll. Essai), Paris, p. 99-131.

Furet François \& Ozouf Jacques, 1977, Lire et écrire. L'Alphabétisation des français de Calvin à Jules Ferry, I, Paris, Les Éditions de Minuit (coll. Le sens commun), Paris, 392 p.

GRUNY Marguerite, 1986, « La bibliothèque pour enfants en France, création et développement de L'Heure joyeuse : l'époque des pionniers » in La Joie par les livres, $\mathrm{n}^{\circ} 110$, p. 47-59.

Hazard Paul, 1967 [1932], Les Livres, les enfants et les hommes, Hatier, Paris, p. 222.

Hoggart Richard, 1970 [1957], La Culture du pauvre, Les Éditions de Minuit (Le sens commun), Paris, p. 423.

Huchet Claire, 1925, «L'Heure joyeuse » in La Nouvelle Éducation, no 33, p. 28-29. 
Kaufman Jean-Claude, 1996, « Portes, verrous et clés : les rituels de fermeture du chez soi » in Ethnologie française, XXVI/2, p. 280-288, https//www.jstor. org/stable/40989655.

Lemaître Henri, 1925, « La Bibliothèque enfantine de la rue Boutebrie, "l'Heure Joyeuse" » in Revue des bibliothéques, $\mathrm{n}^{\circ}$ 1-6, Librairie Ancienne Édouard Champion, p. 28-54.

LERICHe Mathilde, 1956, Préface «Heure du conte » in On raconte... Contes pour les enfants de 5 à 9 ans, Éditions Bourrelier, Paris, p. 3-14.

Naffrechoux Martine, 1987, «Des lecteurs qui s'ignorent » in Bulletin des bibliothéques de France (BBF), no 5, p. 404-419, http://bbf.enssib.fr/ consulter/bbf-1987-05-0404-001.

Résumé : en 1924 est inaugurée L'Heure joyeuse, la première bibliothèque pour enfants créée en France. Les bibliothécaires s'appellent Claire Huchet, Marguerite Gruny et Mathilde Leriche. Formée à L'Heure joyeuse, Jacqueline Dreyfus travaille à la petite bibliothèque de Ménilmontant et dans les années 1930 à la section jeunesse de la bibliothèque de la rue Fessart. Ces pionnières ont laissé des documents qui rendent compte des difficultés rencontrées par les jeunes usagers dans leurs processus d'acculturation à la culture écrite. Il faut, dans la salle de lecture, devenir un lecteur intéressé par les livres et actif dans la communauté des lecteurs ; et lors des heures du conte, il faut cette fois devenir un auditeur attentif à la performance de la conteuse et participer aux mondes imaginaires qui sont offerts de vive voix. Devenir lecteurs, devenir auditeurs suscitent chez les enfants et les adolescents concernés des résistances fortes et des compromis mais aussi, dans certains cas, des adhésions enthousiastes. Ces résistances prennent souvent des formes diverses et réactives d'oralités juvéniles. Ces oralités contestataires ou indisciplinées font ainsi irruption dans le monde de la bibliothèque. Elles en bousculent l'ordre et parfois elles en carnavalisent presque les règles. Quand ces oralités se font joyeuses, elles disent l'anticipation des chances de profit culturel qui se manifestent à travers les régulations entre pairs et la self-discipline. Elles s'expriment aussi dans l'abandon émotif et le silence profond des auditeurs pris dans les rets du récit et sous le charme de la conteuse. Mais ce passage des oralités de résistance aux oralités joyeuses est, pour certains jeunes usagers, un passage toujours renégocié, jamais totalement acquis.

Mots-clefs : anthropologie de la communication, bibliothèque pour enfants, L'Heure joyeuse, Heure du conte, acculturation à l'écrit, lecteurs, auditeurs, oralités de résistances, oralités joyeuses 


\section{Oralities of resistance and joyful oralities}

Abstract: In 1924 L'Heure joyeuse, the first library for children, was created in France. The librarians were Claire Huchet, Marguerite Gruny and Mathilde Leriche. Trained in the framework of L'Heure joyeuse, Jaqueline Dreyfus worked at the small library of the Menilmontant and in the 30s in the children's section of the Fessart Street Library. These pioneers left documents which reveal the difficulties experienced by their young auditors in their initiation into a culture of reading. In the reading room one had to become a reader interested in books and active in the community of readers. And during the storytelling hour, one had to become an auditor attentive to the performance of the storyteller and a participant in the imaginary worlds created by the voice. The process of becoming readers and auditors generated strong resistance in the children and adolescents and necessitated compromises, but sometimes they expressed great enthusiasm. The resistance was often expressed through juvenile forms of orality. These undisciplined manifestations of opposition irrupted in the library, disturbing its order and creating a form of topsy turvy in which the rules were turned upside down. When these forms of orality were joyful they expressed the anticipation of chances for cultural benefit expressed through self-regulation and self-discipline within the group. They could also be seen in the emotional abandon and the deep silence of the auditors caught in the web of the story, under the spell of the storyteller. But this passage from oralities of resistance to oralities of adhesion, was, for certain children, the object of incessant negociation, never totally mastered.

Keywords: anthropology of communication, libraries for children, L'Heure joyeuse, the story hour, acculturation to written texts, readers, auditors, oralities of resistance, joyful oralities

\section{Note sur l'auteur}

Marie-Christine Vinson est membre du CREM, Centre de recherche sur les médiations, à l'Université de Lorraine. Elle travaille en ethnocritique de la littérature (voir site : ethnocritique.com). Elle s'intéresse à la littérature de jeunesse et à la littérature du $\mathrm{XIX}^{\mathrm{e}}$ siècle. Elle a déjà publié dans les CLO en 2007, « Tibili ou l'empire de la littératie » et « Le jeu de l'oye » en 2010. Plus récemment, elle a publié par exemple une étude sur Zola :« Coqueville/ Cocagne » dans les Cahiers Naturalistes [2017] et « La nature morte dans Les Trois Messes basses d'Alphonse Daudet » dans la Revue d'Histoire littéraire de la France [2018]. 